\title{
Neovascular Glaucoma Induced by Peripheral Retinal Ischemia in Neurofibromatosis Type 1: Management and Imaging Features
}

\author{
Francesco Pichi ${ }^{\mathrm{a}}$ Mariachiara Morara ${ }^{\mathrm{b}}$ Andrea Lembo ${ }^{\mathrm{a}}$ \\ Antonio P. Ciardella ${ }^{b} \quad$ Alessandro Meduric ${ }^{c}$ Paolo Nucci ${ }^{a}$ \\ ${ }^{a}$ University Eye Clinic, San Giuseppe Hospital, Milan, ${ }^{b}$ Ophthalmology Unit, Policlinico S. \\ Orsola-Malpighi, Bologna, and ${ }^{\mathrm{C}}$ Ophthalmology Unit, Policlinico G. Martino, Messina, Italy
}

Key Words

Neovascular glaucoma $\cdot$ Neurofibromatosis $\cdot$ Retinal ischemia

\begin{abstract}
Purpose: To report the case of a young patient affected by neurofibromatosis 1 (NF-1) with peripheral retinal ischemia-induced neovascular glaucoma and the peculiar spectral-domain optical coherence tomography (SD-OCT) features. Material and Methods: A 13-year-old boy affected by NF-1, as diagnosed according to established criteria, was referred with a diagnosis of hypertensive uveitis in his left eye. He underwent a complete ophthalmic examination and comprehensive blood work with viral and immunological tests. The case was documented with fluorescein angiography (FA) and SD-OCT. When the intraocular pressure (IOP) of the left eye decreased and the cornea cleared, FA revealed retinal ischemia and leakage from pathologic retinal vessels. SD-OCT revealed foveal hypoplasia secondary to the complete absence of the retinal nerve fiber layer. Results: Peripheral retinal ischemiainduced neovascular glaucoma was diagnosed. The patient underwent Ahmed valve implantation to control his IOP, and subsequent retinal photocoagulation by argon laser and intravitreal bevacizumab injection were performed to control neovascularization. Discussion: Retinal ischemia in NF-1 might lead to neovascular glaucoma: lowering of the IOP with surgical implantation of an Ahmed valve, regression of neovascularization by argon laser panretinal photocoagulation and intravitreal injection of bevacizumab can be a helpful way to control such a complication.
\end{abstract}

Francesco Pichi, MD

University Eye Clinic, San Giuseppe Hospital

Via San Vittore 12

IT-20123 Milan (Italy)

E-Maililmiticopicchio@gmail.com 
Pichi et al.: Neovascular Glaucoma Induced by Peripheral Retinal Ischemia in Neurofibromatosis Type 1: Management and Imaging Features

\section{Introduction}

Neurofibromatosis type 1 (NF-1) is a hereditary disease with systemic vascular ischemic manifestations described in the aorta, brain, kidney and also the eye. Other ocular manifestations in NF-1 are plexiform neurofibromas of the eyelids, Lisch nodules of the iris, congenital glaucoma, optic glioma, retinal astrocytic hamartomas, retinal capillary hemangiomas and microvascular retinal abnormalities [1]. Different vascular retinal abnormalities have been described in NF-1 such as stenosis of small vessels, progressive capillary ischemia and compensatory collateral circulation [2].

We report the case of a 13-year-old boy, affected by NF-1, with a diagnosis of peripheral retinal ischemia-induced neovascular glaucoma and the peculiar spectral-domain optical coherence tomography (SD-OCT) features.

\section{Material and Methods}

A 13-year-old boy affected by NF-1 was referred to our Eye Clinic in January 2011 due to a 1-month history of pain, redness and blurred vision in his left eye, which had been previously diagnosed and treated as hypertensive uveitis. The diagnosis of NF-1 was made in childhood according to established criteria: he had multiple café au lait spots, plexiform neurofibromas of the neck, trunk and limbs, and Lisch nodules of the iris in both eyes. He had no first-degree relatives affected by NF-1.

At our clinic, his best-corrected visual acuity was 20/20 in his right eye and light perception in the left eye. Slit lamp examination in the left eye showed diffuse corneal edema, conjunctival hyperemia and normal anterior chamber depth with pupil mydriasis. No other iris features were visible at that moment nor was the fundus aspect. The intraocular pressure (IOP) was $16 \mathrm{~mm} \mathrm{Hg}$ in the right eye and $45 \mathrm{~mm} \mathrm{Hg}$ in the left eye, by Goldmann applanation tonometry, under maximal medical therapy (acetazolamide $250 \mathrm{mg}$ per os 3 times a day, topical medications such as timolol maleate-dorzolamide fixed combination and topical steroids such as dexamethasone eye drops 6 times a day to control intraocular inflammation).

\section{Results}

The patient was admitted, and therapy was initiated with intravenous mannitol $500 \mathrm{ml}$ twice a day plus maximal topical medications to lower his IOP. The following day, slit lamp examination revealed a clear cornea with iris neovascularization. The IOP was $10 \mathrm{~mm} \mathrm{Hg}$ in the right eye and $32 \mathrm{~mm} \mathrm{Hg}$ in the left eye. Fundus examination of the left eye was now possible and showed optic atrophy and vitreous haze; diffuse retinal vascular changes included dilated and tortuous veins, narrowing of the retinal arterioles and arteriovenous communications.

We performed fluorescein angiography (FA) and SD-OCT using the Heidelberg Spectralis HRA-OCT. FA revealed retinal ischemia and peripheral leakage from pathological retinal vessels and vitreous hemorrhage (fig. 1). SD-OCT of the macula revealed the complete absence of the retinal nerve fiber layer in the posterior pole, and, consequently, no foveal depression was present (fig. 2). Blood biochemistry tests, blood count, erythrocyte sedimentation rate and viral and immunological tests yielded normal results. 
In the end, peripheral retinal ischemia-induced neovascular glaucoma in the left eye was diagnosed. The IOP remained over $40 \mathrm{~mm} \mathrm{Hg}$ for 3 days even with intravenous mannitol 500 $\mathrm{ml}$ twice a day and topical medications to lower his IOP. Because of the failure of medical therapy, he underwent Ahmed valve implantation to control the IOP. On the first postoperative day, the IOP was $28 \mathrm{~mm} \mathrm{Hg}$ in the left eye.

Two weeks after surgery, retinal photocoagulation by argon laser and intravitreal bevacizumab injection under general anesthesia were performed. The final best-corrected visual acuity was count fingers, and the IOP was $26 \mathrm{~mm} \mathrm{Hg}$ in the left eye. No pain or discomfort was reported. We discharged the patient with timolol maleate-dorzolamide fixed combination twice a day.

One month later, FA and SD-OCT were performed. FA revealed no leakage or other retinal vessel abnormalities, and SD-OCT remained unchanged. Slit lamp examination showed a clear cornea, with the tube well positioned in the anterior chamber (fig. 3), complete regression of rubeosis and pupil midriasis. The IOP was $22 \mathrm{~mm}$ Hg by Goldmann applanation tonometry.

\section{Discussion}

Systemic vascular occlusive disease has been previously reported in NF-1 to affect aortic, cerebral, renal, celiac and mesenteric vessels. The pathogenic hypothesis in this case was related to the growth of Schwann cells and dysplasia of arteriolar smooth muscle cells, which led to a progressive narrowing of the vessel and consequent occlusion with secondary fibrous changes [3].

Elgi et al. [4] proposed the hyperproliferation of pericytes and endothelial cells as the cause of retinal vascular occlusion and neovascularization in NF-1. In chronic and slowly progressive arteriolar inadequacy, the veins might not suffer from arterial narrowing in an early phase; in a young patient, progressive occlusion may be due to a more compensative venous system [5]. However, a chronic reduction of arterial supply can lead to progressive retinal ischemia [6].

Ozerdem [7] published a paper about NF-1 gene mutations as a cause of increased proliferation of pericytes and endothelial cells that might enhance the angiogenic response of the retina to the ischemia. Moreover, loss of neurofibromin expression in endothelial cells could affect the interactions between endothelial cells and pericytes with a consequently abnormal proliferation of both these cells and final vascular occlusion. Elgi et al. [4] reported a case very similar to ours, emphasizing the unilateral appearance of the disease. The mechanism of this unilaterality, which we observed as well, is not fully understood but is reported in other organs too, such as the kidney, brain and large vessels of the heart; it is recognized as a characteristic feature of ischemia in NF-1. Furthermore, we postulate that in our patient arteriolar occlusion associated with microvascular alterations and peripheral retinal ischemia could be the cause of neovascular glaucoma, even if the angiogram did not register arteriovenous communication between perfused and nonperfused retinas. A vitreous opacity previously noted by Elgi et al. [4] was supposedly the aftermath of a vitreous hemorrhage.

One characteristic feature of the SD-OCT was the absence of the foveal depression, which we related to a glaucomatous optic nerve and complete atrophy of the retinal nerve fiber layer. As has been previously reported, we also associated the use of intravitreal bevacizumab and argon laser panretinal photocoagulation with better control of neovascularization. 
Case Reports in

Ophthalmology

\begin{tabular}{l|l}
\hline Case Rep Ophthalmol 2013;4:69-73 \\
\hline DOI: 10.1159/000350956 & $\begin{array}{l}\text { @ 2013 S. Karger AG, Basel } \\
\text { www.karger.com/cop }\end{array}$ \\
\hline
\end{tabular}

Pichi et al.: Neovascular Glaucoma Induced by Peripheral Retinal Ischemia in Neurofibromatosis Type 1: Management and Imaging Features

\section{References}

1 Destro M, D’Amico DJ, Gragoudas ES, Brockhurst RJ, Pinnolis MK, Albert DM, Topping TM, Puliafito CA: Retinal manifestations of neurofibromatosis. Diagnosis and management. Arch Ophthalmol 1991;109:662666.

-2 Karadimas P, Hatzispasou E, Bouzas EA: Retinal vascular abnormalities in neurofibromatosis type 1. J Neuroophthalmol 2003;23:274-275.

-3 Mori F, Kawai M, Sato E, Igarishi S, Hikichi T, Yoshida A: Branch retinal vein occlusion in a Japanese patient with neurofibromatosis 1. Jpn J Ophthalmol 2001;45:634-635.

-4 Elgi U, Berker N, Teke MY, Simsek T, Ozdal P: Unusual association of peripheral retinal ischemia-induced neovascular glaucoma and neurofibromatosis type 1.J Pediatr Ophthalmol Strabismus 2010;47:e1-e3.

-5 Thölen AM, Messmer EP, Landau K: Peripheral retinal vascular occlusive disorder in a young patient with neurofibromatosis 1. Retina 1998;18:184-186.

6 Moadel K, Yannuzzi LA, Ho AC, Ursekar A: Retinal vascular occlusive disease in a child with neurofibromatosis. Arch Ophthalmol 1994;112:1021-1023.

7 Ozerdem U: Targeting neovascular pericytes in neurofibromatosis type 1. Angiogenesis 2004;7:307-311.

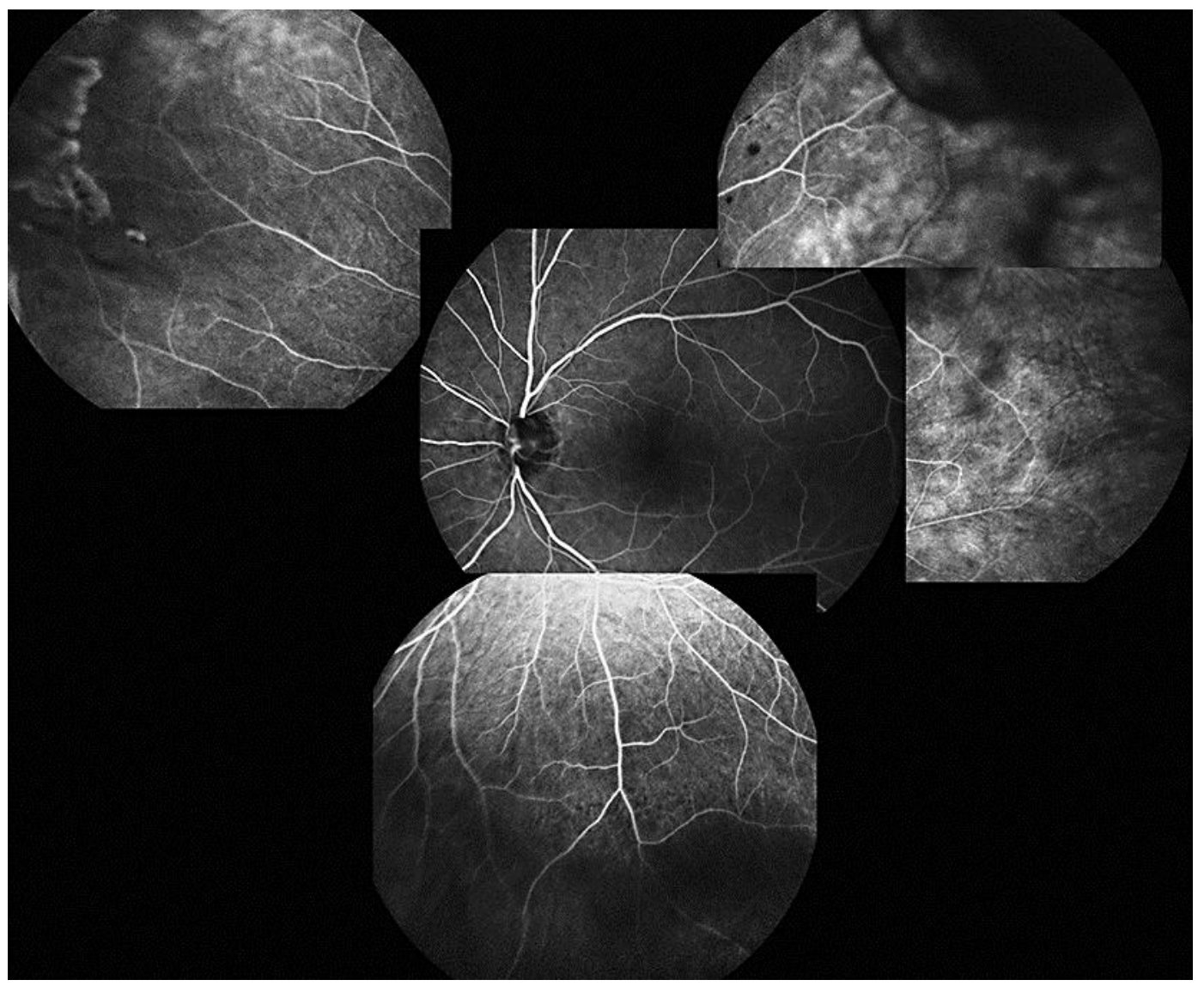

Fig. 1. Composite FA, left eye. The optic nerve head is atrophic and hypofluorescent. In the peripheral retina, significant leakage from pathological vessels and ischemia are evident. 
Case Reports in

Ophthalmology

\section{Case Rep Ophthalmol 2013;4:69-73}

DOI: 10.1159/000350956

chi et al:: Neovascular Glaucoma Induced by Peripheral Retinal Ischemia in

Neurofibromatosis Type 1: Management and Imaging Features

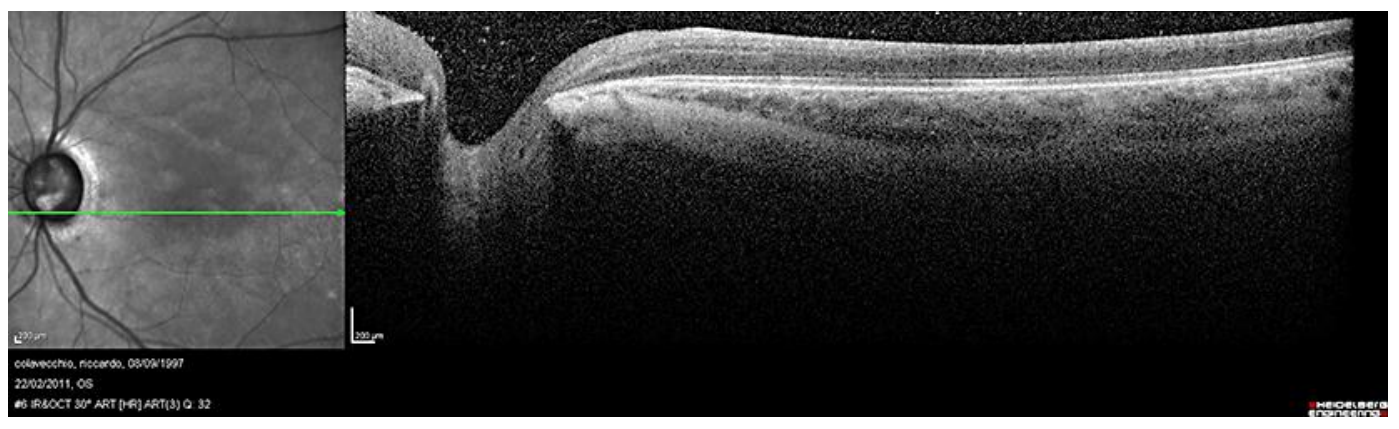

Fig. 2. OCT demonstrates the complete absence of the retinal nerve fiber layer in the macula and, consequently, no foveal depression.

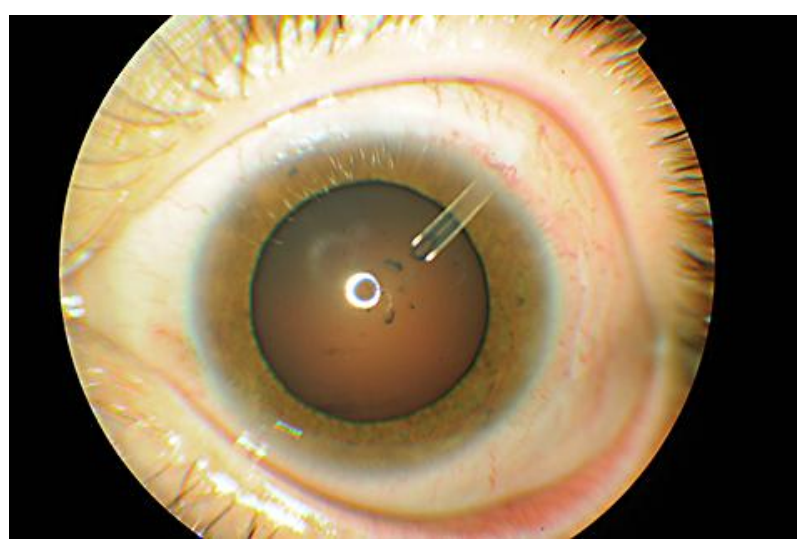

Fig. 3. One month after surgery the cornea is clear, no rubeosis can be seen and the tube is well positioned in the anterior chamber. 